\title{
Patientensicherheit in der Endoskopie: Prävention und Management von kritischen Ereignissen bei der Sedierung
}

\author{
Marcus Rall Stephanie Oberfrank \\ InPASS, Institut für Patientensicherheit und Teamtraining, Reutlingen, Deutschland
}

\section{Schlüsselwörter \\ Crisis Resource Management, CRM - Human Factors, HFs . Systemsicherheit . Simulationsteamtraining . "Critical Incident Reporting»-System, CIRS}

\section{Zusammenfassung}

Hintergrund: Die Verbesserung der Patientensicherheit in der Endoskopie ist nicht einfach und nicht von heute auf morgen zu realisieren. Der größte Anteil an schwerwiegenden Zwischenfällen bei der Endoskopie ist auf die Sedierung zurückzuführen. Methode: Anwendung der Inhalte der S3-Leitlinie sowie aktueller Kenntnisse zur Erhöhung der Patientensicherheit. Ergebnisse: Es ist selbstverständlich, dass die gemäß der aktuellen S3-Leitlinie geforderten Personal-, Struktur- und Prozessvoraussetzungen bei einer Sedierung in der Endoskopie auch bei Personalknappheit kompromisslos eingehalten werden. Risikopatienten müssen zuverlässig identifiziert und dann von entsprechend (intensivmedizinisch) erfahrenem Personal während endoskopischer Eingriffe betreut werden. Dennoch gilt: Fehler sind in komplexen Systemen zu erwarten und insofern «normal». Aber wir müssen aus Fehlern lernen. Hierfür bieten moderne softwarebasierte "Critical Incident Reporting"-Systeme (CIRS) gute Voraussetzungen. 70\% der medizinischen Fehler beruhen nicht auf mangelndem Fachwissen, sondern auf Problemen im Bereich der Human Factors (HFs). Crisis Resource Management (CRM) ist ein effektives Tool gegen HF-bedingte Fehler und sollte in Zukunft von allen beherrscht werden. Schlussfolgerungen: Der aktuelle Goldstandard für HF/CRM-Training ist der Einsatz von realitätsnahen und modernen Simulationsteamtrainings, die von speziell geschulten Instruktoren durchgeführt werden. Um die Patientensicherheit nachhaltig zu erhöhen, sind die Autoren der Auffassung, dass Simulationstrainings zu Sedierungen und Zwischenfällen in der Endoskopie mindestens zweimal pro Jahr für jeden medizinischen Mitarbeiter bei Sedierungen in der Endoskopie durchgeführt werden sollten.

\section{KARGER \\ Fax +497614520714 \\ Information@Karger.com}

www.karger.com (c) 2013 S. Karger GmbH, Freiburg

1662-6664/13/0293-0150\$38.00/0

Accessible online at:

www.karger.com/vim
Keywords

Crisis Resource Management, CRM - Human factors, HFs .

System safety - Simulation team training .

Critical Incident Reporting System, CIRS

\section{Summary}

Patient Safety in Endoscopy: Prevention and

Management of Critical Incidents Related to Sedation

Background: Improvement of patient safety in endoscopy is neither simple nor easily accomplished from today to tomorrow. Most of the severe critical incidents occurring during endoscopy are related to sedation. Method: Application of the contents of the S3 guidline and of current knowledge on the increase of patient safety. Results: Provision of the necessary personnel and structural and procedural requirements during sedation in endoscopy as requested by the $\mathrm{S} 3$ guidelines is a self-evident obligation, even if there is a shortage of staff. During sedation in endoscopy, patients at risk need to be clearly identified and dealt with by (in intensive care) experienced personnel. Nevertheless, errors in complex systems are to be expected and therefore have to be considered as 'normal'. Still, we need to learn from errors. For this purpose, software-based Critical Incident Reporting Systems (CIRSs) provide excellent conditions. $70 \%$ of the medical errors are not due to a lack of medical knowledge but to 'human factors' (HFs). Crisis Resource Management (CRM) is an effective tool against HF-related errors and should therefore be well employed by everybody. Conclusions: For this purpose, the current gold standard is the application of realistic and modern simulation team trainings carried out by particularly trained instructors. In order to sustainably improve patient safety, the authors recommend compulsory simulation team trainings for all healthcare professionals at least two times a year. 


\section{Einleitung}

Über 50\% der ernsten Komplikationen bei endoskopischen Eingriffen sind unerwünschten Wirkungen der (Analgo-)Sedierung zuzuschreiben [1, 2]. Nicht selten führen Zwischenfälle im Zusammenhang mit der Sedierung zu schwerwiegenden, manchmal lebensbedrohlichen Situationen. Während die Sedierungsfrequenz bei endoskopischen Eingriffen in Deutschland und der Schweiz Mitte der 1990er-Jahre bei etwa 9\% lag [3-5], wird sie heutzutage mit bis zu 87\% angegeben [5, 6]. AuBerdem wächst die Zahl an Risikopatienten. Damit kommen Ereignisse, die früher relativ selten waren, heute häufiger vor.

Die grundsätzliche Vermeidbarkeit dieser Zwischenfälle und die enorm ansteigende Zahl an Endoskopien unter Sedierung sprechen für einen dringenden Handlungsbedarf.

Studien zeigen, dass hinter der Entstehung von kritischen Ereignissen und medizinischen Zwischenfällen in der Regel nicht einzelne Fehler stehen, sondern vielmehr eine oft unglückliche Verkettung von multiplen Ereignissen, Begleitfaktoren und latenten Fehlern im System. Zudem entstehen medizinische Zwischenfälle in bis $\mathrm{zu} 70 \%$ der Fälle nicht aufgrund mangelnden fachlichen Wissens oder Könnens, sondern werden durch sogenannte Human Factors (HFs) hervorgerufen. Die Prävention und das Management von kritischen Ereignissen und medizinischen Zwischenfällen kann durch Schulungen im Bereich HF und Fehlerentstehung sowie in der Anwendung von Crisis Resource Management (CRM) in komplexen Situationen erreicht werden. Hierbei handelt es sich um Themenbereiche, die in der bisherigen medizinischen Ausund Weiterbildung keine oder lediglich eine sehr untergeordnete Rolle gespielt haben und in modernen, realitätsnahen Simulationsteamtrainings erlernt und trainiert werden müssen.

Stürze von sedierten oder narkotisierten Patienten von Behandlungsliegen oder OP-Tischen kommen leider immer wieder vor und gehören damit zu den bekannten «klassischen» Zwischenfällen in der Endoskopie (Kasten 1). Besonders brisant ist in diesem Fall auch, dass diese Ereignisse klar theoretisch vermeidbar sind und immer zur Haftung des Arztes oder der Institution führen, da der Patient sich auf den Schutz während einer Sedierung verlassen können muss (fahrlässig oder grob fahrlässig).

In der Praxis ist es hingegen nicht leicht, diese Zwischenfälle zuverlässig zu verhindern (Kasten 2): Sedierte Patienten sind unberechenbar, machen einen «friedlichen» Eindruck, drehen sich aber im nächsten Moment mit großer Kraft, zum Teil auch verwirrt und desorientiert um. Eine Sekunde der Unaufmerksamkeit oder kurze Abwesenheit vom ungesicherten Patientenbett kann für einen schweren Zwischenfall (z.B. schwere innere Blutungen, Pneumothorax, Schädelhirntrauma, multiple Extremitätenfrakturen usw.) ausreichen.

Ist es zum Sturz gekommen, sollte man dies keinesfalls «herunterspielen». Der Patient muss umfassend informiert sowie initial und anschließend engmaschig untersucht werden (Abdomenultraschall, gegebenenfalls Computertomographie (CT), Röntgen der Extremitäten usw.).
Die akzidentielle Überdosierung von Propofol (z.B. durch Applikation eines Propofolbolus) mit anschließendem Atemstillstand und eventuell resultierender Hypoxie des Patienten gehört zu den typischen und schwerwiegendsten Zwischenfällen in der Endoskopie (Kasten 3). Auch diese Art von Zwischenfall ist vermeidbar, was im Folgenden dargestellt werden soll.

Kasten 1. Fall 1: Der «Sturz von der Behandlungsliege unter Sedierung»

Nach Beendigung der Gastroskopie unter Propofolsedierung ist der Arzt mit der Dokumentation beschäftigt. Die Pflegekraft, die für die Sedierung zuständig ist, räumt die Untersuchungsmaterialien weg, da die zweite Pflegekraft heute krankheitsbedingt fehlt. Beim Aufräumen bewegt sie sich einige Meter vom Patientenbett weg. In diesem Moment macht der Patient, der inzwischen die ersten Aufwachreaktionen zeigt, eine schnelle Rollbewegung auf der Liege und stürzt, bevor Arzt oder Pflegekraft hinzueilen können, hart auf den Boden. Neben einer Platzwunde am Kopf zieht sich der ohne Schutzreflexe sediert gefallene Patient eine zweifache Rippenfraktur zu.

Kasten 2. Prävention von Stürzen von der Liege von sedierten Patienten

- Bei sedierten Patienten muss immer eine Person unmittelbar am Patientenbett stehen. Patienten in Sedierung dürfen nie allein gelassen werden, auch nicht «für 2 Sekunden, um mal kurz etwas zu holen, abzustellen» usw. Dies muss das Team verstehen («Ich kann grad nicht weg») und mittragen («Bleiben Sie bitte da stehen, ich hole das Gerät oder schicke jemand anderes»). Bei Kindern gilt: immer eine Hand am Kind!

- Ein einzelner Gurt, egal ob an Bauch, Becken oder Oberschenkel, reicht nicht aus. Außerdem sollten z.B. Klettgurte regelmäßig erneuert werden, da die Haltekraft nachlässt.

- Falls man beim Abklingen der Sedierung vom Bett/von der Liege des Patienten weggeht, muss eine entsprechend leistungsfähige Sicherung zum Einsatz kommen (Bettgitter beidseits und auf voller Breite oder Ähnliches).

- Das Umlagern von Patienten unter anhaltendem Sedierungseinfluss (Liege/Bett) muss immer mindestens zu zweit erfolgen. $\mathrm{Zu}$ leicht rutscht der Patient auf der Seite herunter, an der gerade niemand steht. Alle Bremsen immer zu!

- Ist ein Patient trotz aller Vorsichtsmaßnahmen von der Liege gefallen, muss dies ernst genommen werden. Eine verzögerte Diagnostik und Therapie sind völlig inakzeptabel. Der Patient muss über den Sturz und etwaige Folgen informiert werden.

Kasten 3. Fall 2: Hypoxie nach akzidentieller Gabe eines Propofolbolus

Adipöser Patient während Koloskopie. Propofol wird durch eine speziell geschulte Pflegekraft per Spritzenpumpe verabreicht. Weil die Sedierung an einer schwierigen Stelle der Endoskopie nicht ausreicht, ordnet der Arzt eine sofortige tiefere Sedierung an. Die Pflegekraft verabreicht einen Bolus Propofol, der Arzt ist zufrieden. 30 Sekunden später schlägt die Pflegekraft Alarm: «Der Patient atmet nicht mehr!» Die Pflegekraft versucht, den Patienten mit Maske und Beutel zu beatmen. Dies gelingt nicht gut. Der Patient wird zyanotisch und erbricht zudem. Der Arzt ruft das Reanimationsteam, bei dessen Eintreffen der Patient bereits tief zyanotisch und aufgrund der prolongierten Hypoxie bradykard ist. Der Patient erholt sich auf der Intensivstation gut, zeigt aber anhaltende Konzentrationsstörungen als Zeichen einer stattgefundenen zerebralen Hypoxie. 


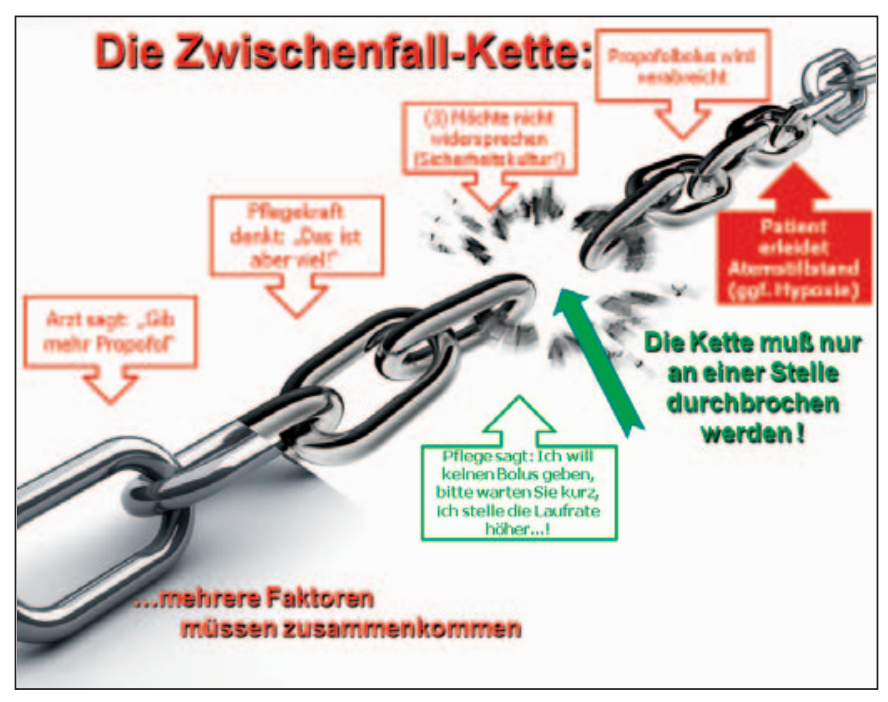

Abb. 1. Entstehung eines Zwischenfalls am Beispiel der Fehlerkette: Die Verknüpfung mehrerer Kettenglieder führt dazu, dass am Ende der Kette ein Patient zu Schaden kommt. Die gute Nachricht: Es ist nur eine einzige Intervention nötig, um ein derartig kritisches Ereignis abzuwenden.

\section{Entstehung von Zwischenfällen}

Untersuchungen belegen, dass nur selten isolierte Faktoren oder einzelne Personen Auslöser von Zwischenfällen sind. Die Ursachen liegen vielmehr in vielfältigen Defiziten des anfälligen «Systems Patientensicherheit» selbst [7-10], das in medizinischen Einrichtungen im Gegensatz zu vielen anderen Hochsicherheitsindustrien (sogenannten High-Reliability Organizations, HROs) wie beispielsweise der Luft- und Raumfahrt oder der Großchemie nicht auf Sicherheit optimiert ist.

Viele Zwischenfälle in der Medizin haben ihre Ursachen in technischen und organisatorischen Rahmenbedingungen innerhalb des Systems (sogenanntes Sick-Organization-Syndrom) [10]. In der Luftfahrt wurde nachgewiesen, dass die meisten Piloten gleich gehandelt hätten wie die Piloten in den Unglücksmaschinen; es handelte sich also um systematische Ausbildungsprobleme [11]. In der Medizin ist dies bestimmt ähnlich. Leider ist die Suche nach dem «Schuldigen» nach einem bedrohlichen Zwischenfall, gemäß der sogenannten Schuldkultur («Culture of Blame»), immer noch weit verbreitet. Um die Patientensicherheit systematisch zu erhöhen, ist es wichtig, das Augenmerk auf die Frage nach dem «Warum ist es passiert?» zu richten, nicht auf das «Wer ist dafür verantwortlich?».

\section{Die Ursachenkette des Zwischenfalls durchtrennen}

Während die Entstehung eines Zwischenfalls, wie oben erwähnt, erst aus der Verkettung zahlreicher Faktoren resultiert und im Einzelfall schwer vorherzusehen ist, bedarf es zur Ab- wendung eines Patientenschadens oft nur einer einzigen Intervention (Abb. 1). Die gute Nachricht ist also: Man muss nur ein Kettenglied durchtrennen, um den Schaden von diesem Patienten abzuwenden. Das Erkennen der Gefahren und effektive Intervenieren (Durchtrennen der Zwischenfallkette) kann und sollte geübt werden.

\section{Ursachen von Zwischenfällen meist im Bereich Human Factors}

Ungefähr $70 \%$ aller Zwischenfälle haben ihre Ursachen nicht in mangelndem medizinischen Fachwissen, sondern im Bereich der HFs. HFs werden oft auch als «non-technical skills» bezeichnet. Analog der Medizin liegt die Größenordnung der HF-assoziierten Zwischenfälle auch in anderen Hochsicherheitsorganisationen ähnlich hoch [9, 12].

Unter dem Begriff der HFs wird eine Vielzahl von Fähigkeiten zusammengefasst, die menschliches Verhalten und Leistungsfähigkeit in komplexen Situationen beeinflussen. Generell unterscheidet man sowohl positive als auch negative HFs. Positive HFs unterstützen die Anwendung von Sicherheitsmaßnahmen in komplexen Situationen, wohingegen negative HFs diesen Effekt limitieren können. Tabelle 1 zeigt die Aufteilung der verschiedenen Aspekte von HFs. In Teamtrainings gilt es, diese Fähigkeiten zu schärfen und für möglicherweise resultierende Gefahren zu sensibilisieren.

\section{Risiken und mögliche Komplikationen der (Analgo-) Sedierung bei der Endoskopie}

Treten bei endoskopischen Eingriffen Komplikationen auf, sind diese in mindestens $50 \%$ der Fälle unerwünschten Wirkungen der Sedierung zuzusprechen $[1,2]$.

Heutzutage sind die früher verwendeten Benzodiazepine vielfach durch das kurzwirksame Hypnotikum Propofol abgelöst worden. Allerdings ist die Sedierung mit Propofol eine Gratwanderung, denn Propofol ist ein Narkosemittel. Bei zu hoher oder zu schneller Medikamentenapplikation geschieht der Übergang zur Allgemeinanästhesie manchmal unerwartet und rasch. Dabei sind nicht nur die Atemdepression und die Ausschaltung der Schutzreflexe (Aspiration) problematisch, auch anhaltende Blutdruckabfälle mit eventuell resultierender Koronarischämie (diastolischer Blutdruck!) können Probleme bereiten. Dann wäre ein voll ausgebildeter Anästhesist erforderlich. Obwohl Propofol zahlreiche Vorteile für die Sedierung aufweist, liegt ein großer Nachteil von Propofol darin, dass mit einzelnen Dosen eine weitaus stärker ausgeprägte Sedierungstiefe als beabsichtigt induziert werden kann. Wichtig ist auch, dass kein Antagonist zur Verfügung steht.

Tabelle 2 gibt einen Überblick über Risiken und mögliche Komplikationen einer (Analgo-)Sedierung. 
Tab. 1. Aufteilung von HFs nach verschiedenen Aspekten

\begin{tabular}{|c|c|c|}
\hline Individuelle und kognitive HFs (Gehirn) & Interaktive und kooperative HFs (Team) & $\begin{array}{l}\text { Direkt leistungsbestimmende } \\
\text { Faktoren (Körper) }\end{array}$ \\
\hline $\begin{array}{l}\text { Dynamisches Situationsbewusstsein: z.B. bewusste } \\
\text { Evaluation der Situation, «einen kühlen Kopf } \\
\text { bewahren», den Überblick behalten usw. }\end{array}$ & $\begin{array}{l}\text { Optimale Kommunikationsstruktur (verbal und } \\
\text { nonverbal): z.B. Ansprache mit dem Namen, } \\
\text { «Close-loop-Kommunikation» }\end{array}$ & Müdigkeit \\
\hline $\begin{array}{l}\text { Dynamische Prioritätensetzung und kritische } \\
\text { Reevaluation: z.B. bei Verschlechterung der } \\
\text { Situation }\end{array}$ & $\begin{array}{l}\text { Teamwork: kritische, aber konstruktive Anmerkungen, } \\
\text { klare Führungsrolle, aktive Teammitglieder }\end{array}$ & Lärm \\
\hline Akzeptieren der Unfähigkeit zum Multitasking & $\begin{array}{l}\text { Optimale Verteilung der Arbeitslast; Nutzung aller } \\
\text { Ressourcen; rechtzeitig Hilfe holen }\end{array}$ & Krankheit \\
\hline $\begin{array}{l}\text { Task Management: Organisation der Arbeitsaufgaben } \\
\text { in gut strukturierte Teilkomponenten mit } \\
\text { eingebauten Kontrollen }\end{array}$ & $\begin{array}{l}\text { Übereinstimmende mentale Modelle: Ziehen alle } \\
\text { Teammitglieder am gleichen Strang? Ist jedem die } \\
\text { Diagnose und Behandlungsstrategie bewusst? }\end{array}$ & mentaler Druck \\
\hline $\begin{array}{l}\text { Durchsetzungsvermögen/Beharrlichkeit: bei relevanten } \\
\text { Fragen, Details und Vorschlägen Beharrlichkeit } \\
\text { zeigen }\end{array}$ & Absprachen/Briefings und Debriefings & beruflicher/privater Stress \\
\hline $\begin{array}{l}\text { Mensch/Maschine-Schnittstelle: z.B. Kenntnis des } \\
\text { Arbeitsumfelds und der Geräte }\end{array}$ & $\begin{array}{l}\text { Gemeinsame Ziele: Teilt das Team gemeinsame Werte } \\
\text { und Vorstellungen, z.B. in Bezug auf die «Culture of } \\
\text { Safety»? Werden diese auf den und durch die } \\
\text { Führungsebenen unterstützt? }\end{array}$ & Alter, Erfahrung \\
\hline Kritische Selbstreflexion der Teammitglieder? & & Sehkraft, Gehör, Hunger usw. \\
\hline
\end{tabular}

Tab. 2. Überblick über die Risiken und möglichen Komplikationen einer (Analgo-)Sedierung

Hypoxie ( $\leq 90 \% \mathrm{SpO}_{2}$ ) durch Atemdepression oder Apnoe

Übelkeit/Erbrechen mit Aspiration (Aspirationspneumonie mit hoher Letalität)

Arterielle Hypotonie/myokardiale Ischämie

Herz-Kreislauf-Stillstand

Lagerungsschäden

Sturz von der Liege/vom Bett

Allergische Reaktionen

Tachykardie/bradykarde Herzrhythmusstörungen

Infektionsrisiko z.B. durch mehrfache Wiederverwendung der Medikamentenampulle/Infusionssysteme

Paradoxe Arzneimittelwirkungen

Bakteriämien bis hin zur Sepsis

Pankreatitiden (bei Verwendung von Propofol)

Eventuell «Propofol-Infusions-Syndrom» mit Rhabdomyolyse (bei Verwendung von Propofol, bisher jedoch nur bei Langzeitapplikation aufgetreten)

\section{Präventionsmaßnahmen zur Verhinderung von kritischen Ereignissen in der Endoskopie}

Im November 2008 wurde die erste S3-Leitlinie «Sedierung in der gastrointestinalen Endoskopie» (AWMF-Register-Nr. 021/014) in der Zeitschrift für Gastroenterologie veröffentlicht [5]. Die Einhaltung dieser Leitlinien ist ein wesentlicher
Schritt, die Anzahl kritischer Ereignisse sowie medizinischer Zwischenfälle merklich zu senken und dadurch die Patientensicherheit zu erhöhen.

Um die Sicherheit bei der Sedierung während endoskopischer Eingriffe zu gewährleisten und die Patientensicherheit nachhaltig zu verbessern, ist ein professionelles und sicheres Vorgehen geboten. Dies erfordert neben einer guten Aus- und Fortbildung im Umgang mit Sedativa/Hypnotika, dem passenden medizinischen und technischen Equipment und dem kooperativen Zusammenspiel aller beteiligten Mitarbeiter vor allem eine zuverlässige Identifikation von Risikopatienten (Tab. 3) und deren sorgfältiges Management (keine Endoskopie von Hochrisikopatienten in der Praxis oder ambulant, Hinzuziehung von mehr und erfahrenem Personal (Anästhesie)).

\section{Crisis Resource Management - Management und Prävention von kritischen Ereignissen}

HROs wissen, dass (menschliche) Fehler unmöglich eliminiert werden können, und akzeptieren aus diesem Grund die Präsenz der menschlichen Fehlbarkeit im Gefüge der Systemsicherheit $[14,15]$. Demzufolge sind deren Sicherheitssysteme so aufgebaut, dass es einem einzelnen Menschen «nicht möglich ist», einen derart folgenreichen Fehler zu begehen, dass ein Patient unvermeidbar Schaden erleidet. Hollnagel et al. [16] bezeichnen diesen Sachverhalt als «Resilience». 
Abb. 2. CRM-Karte: Die 15 CRM-Prinzipien nach Rall und Gaba [7]. Die 15 CRM-Sätze sind als einfach anzuwendende Gedankenstützen gedacht, um HF-Stolperfallen zu umgehen und die Zuverlässigkeit menschlicher Leistung in komplexen Situationen zu optimieren. Die aufgelisteten CRM-Prinzipien sind bewusst in sich wiederholendem Charakter konzipiert, um zu gewährleisten, dass einerseits sämtlichen Aspekten der HFs entgegengewirkt werden kann und andererseits verschiedene Personen auf unterschiedlichen Ebenen die Entstehung von kritischen Ereignissen verhindern können.

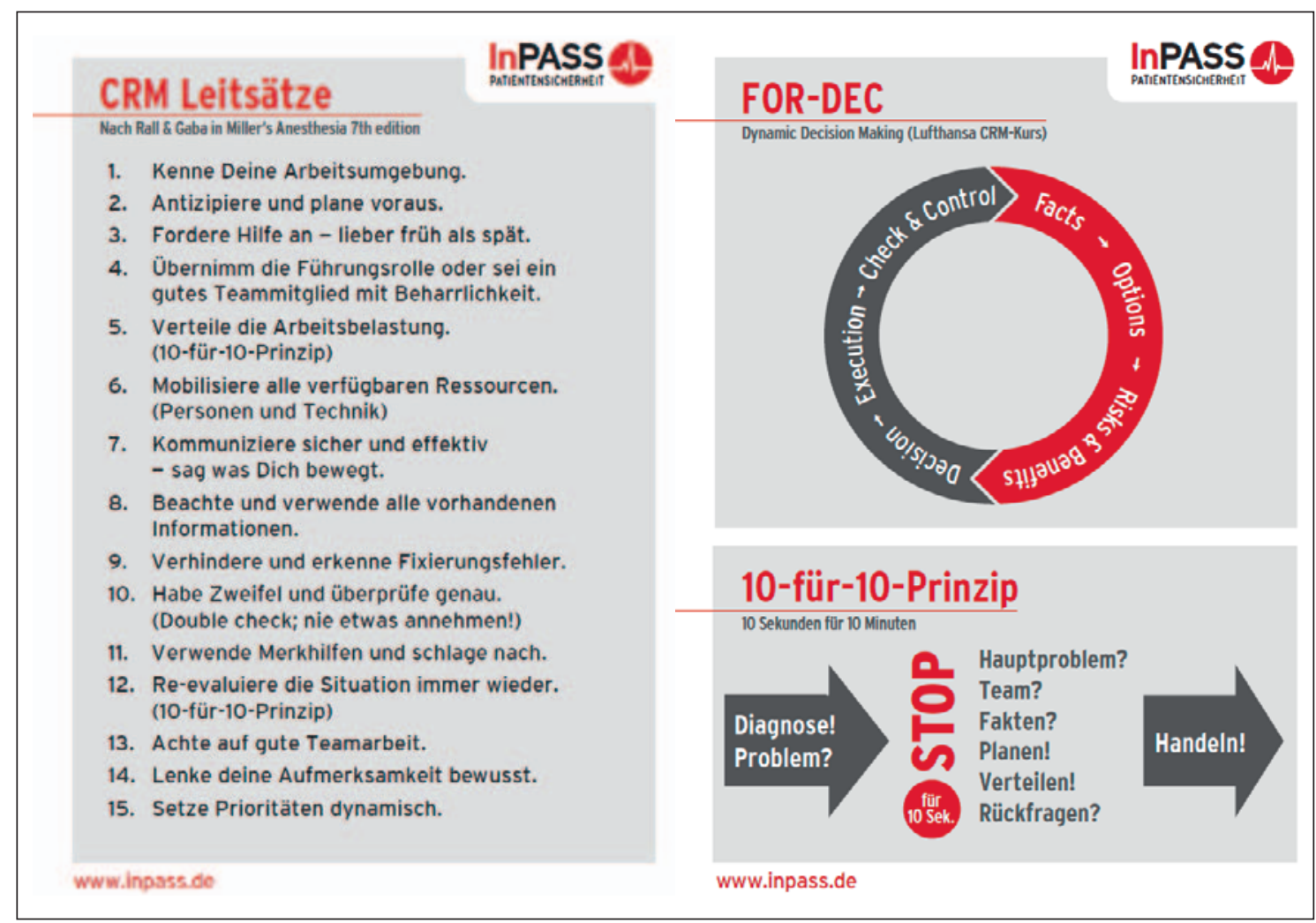

Tab. 3. Identifikation von Risikopatienten für die Durchführung von endoskopischen Eingriffen unter (Analgo-)Sedierung (modifiziert nach [1, 5, 13]) (Empfehlungsgrad A, Evidenzstärke 5, § 1.5 [5])

Risikopatienten sind Patienten mit einem oder mehreren der folgenden Merkmale

Erhebliche Adipositas (Body-Mass-Index > 30)

Schlafapnoesyndrom/Schnarchen/Stridor

Bekannte schwierige Beatmung

Lungenerkrankungen mit partieller oder globaler Ventilationsstörung

Unmöglichkeit einer verbalen Kommunikation

ASA-Klasse $>$ II

NYHA > II, instabile Angina pectoris, KHK

Herzklappenerkrankungen/-ersatz

Dekompensierte Herzinsuffizienz

Leber-/Niereninsuffizienz

Gerinnungsstörungen

Alter $>80$ Jahre

Anatomische Normabweichungen im Bereich der oberen Luftwege

Atemwegsbehinderungen (z.B. Tumoren)

Erhöhtes Aspirationsrisiko

Schwierige endoskopische Eingriffe

Medikamenten-/Alkohol-/Drogenabusus

Mangelnde Kooperationsfähigkeit

ASA = American Society of Anaesthesiologists; NYHA = New York

Heart Association; KHK = koronare Herzkrankheit.

Ein wesentlicher Bestandteil solcher Sicherheitskonzepte ist das CRM. Hierbei handelt es sich um ein effektives Tool, mit dem alle Teammitglieder durch die Anwendung verschiedener Interventionsmaßnahmen sowohl auf der individuell- kognitiven als auch auf der kommunikativ-kooperativen HFEbene (Tab. 1) die Entstehung kritischer Ereignisse unterbinden können. Im Falle eines möglicherweise bevorstehenden kritischen Ereignisses kann CRM jedoch auch dazu genutzt werden, (weiteren) Schaden wirkungsvoll abzuwenden. Daher gilt: CRM ist präventiv, proaktiv und reaktiv!

Ursprünglich kommt CRM aus der zivilen Luftfahrt und wird diesbezüglich auch oft als Crew oder Cockpit Resource Management bezeichnet [17]; CRM wurde für die Bedürfnisse der Medizin bereits vielfach adaptiert. Abbildung 2 zeigt die 15 weiterentwickelten CRM-Hauptprinzipien nach Rall und Gaba [7].

Die vorgestellten CRM-Prinzipien dienen als Vorgehensweisen sowohl für das Individuum als auch für das gesamte Team und verfolgen das Ziel, die Systemsicherheit in komplexen Situationen zu erhöhen. Somit können Stolperfallen durch negative HFs effektiv eliminiert und kritische Ereignisse verhindert werden. Durch die bewusst gewählte Redundanz der verschiedenen Prinzipien, die sich wie ein Sicherheitsnetz mehrfach überlappen, werden sämtliche Elemente der HFs aus Tabelle 1 mehrfach abgedeckt.

\section{Realitätsnahe, moderne Konzepte für Simulations- teamtrainings}

Die CRM-Prinzipien sind nicht zum Auswendiglernen gedacht - sie müssen explizit im Arbeitsalltag Anwendung finden, um auch in komplexen (Notfall-)Situationen beherrscht 
zu werden. Um dies zu gewährleisten, sollten realitätsnahe, moderne Simulationsteamtrainings durch in CRM ausgebildete Instruktoren regelmäßig durchgeführt werden [18-22]. Simulationstrainings, die ohne Fokus auf HFs und CRM durchgeführt werden, entsprechen nicht mehr dem aktuellen Stand der Wissenschaft. Im Gegensatz zu den bisherigen Notfalltrainings basieren moderne Simulationsteamtrainings auf fundierten Konzepten der Erwachsenenbildung. Diese ermutigen die medizinischen Mitarbeiter durch Unterstützung vonseiten der Instruktoren zur Selbstreflexion in kollegialer Atmosphäre und induzieren somit tiefgreifende Lernprozesse. Im Mittelpunkt jedes Simulationstrainings steht das audio-/videoassistierte Debriefing, die Szenarien-Nachbesprechung. Hier findet im Sinne der weiter oben beschriebenen «Safety Culture» zusammen mit den Instruktoren die Analyse des Szenarios und dadurch der eigentliche Lernprozess statt [23]. Es geht dabei nicht darum, Wer? vermeintlich Was? im Simulationsszenario falsch gemacht hat, sondern Warum? verschiedene Aspekte des Simulationsszenarios weniger gut oder aber auch besonders erfreulich verliefen. Auf diese Art und Weise kann das beobachtete Verhalten systematisch analysiert und von den Teilnehmern verstanden werden. Im Team werden darauf aufbauend durchführbare Alternativen erarbeitet, um zukünftig negative Handlungsweisen zu vermeiden und im Gegenzug positives Verhalten zu stärken.

Bei der Durchführung von Simulationsteamtrainings sollte darauf geachtet werden, dass die personelle Trainingszusammensetzung der realen Arbeitsumgebung entspricht. Das Team sollte initial «en bloc» trainiert werden, d.h., dass das gesamte Team innerhalb kürzester Zeit geschult wird. Mit dieser Methode ist der anfängliche Lerneffekt in der klinischen Praxis größer und hält länger an. Wichtig ist jedoch vor allem: Beginnen Sie jetzt! Warten Sie nicht auf den großen Simulator oder das Hightech-Video-/Audio-Equipment, denn jedes Training mit einem guten Instruktor ist besser als kein Training!

\section{"Critical Incident Reporting»-Systeme - Lernen von anderen und für andere}

Fehler sind normal. Aber wir müssen aus Fehlern lernen [24]! Kritische Ereignisse sind Vorläufer von schwerwiegenden Zwischenfällen. Ziel ist es daher, diese zu erkennen und $\mathrm{zu}$ vermeiden, bevor etwas passiert. Systeme zur Erfassung und Analyse dieser kritischen Ereignisse, sogenannte «Critical Incident Reporting»-Systeme (CIRS), können sowohl klinikintern als auch institutionsübergreifend einen bedeutenden Beitrag leisten, Systemschwachstellen zu enttarnen, um durch die Etablierung geeigneter Maßnahmen die Patientensicherheit präventiv zu erhöhen [25, 26]).

Im neuen Patientenrechtegesetz werden CIRS für jedes Krankenhaus verpflichtend.
Kasten 4. Das darf im Umgang mit sedierten Patienten heute nicht mehr passieren

- Durchführung der Sedierung von nicht speziell geschultem Personal (vergleiche S3-Leitlinie)

- Sedierungspersonal muss nebenher andere Aufgaben erfüllen, kann sich folglich nicht $100 \%$ ig dem sedierten Patienten zuwenden

- Unzureichendes Monitoring für die Sedierung (Atemfrequenz!)

- Applikation der sedierenden Medikamente durch den Untersucher

- Fehlendes oder insuffizientes Notfall-Teamtraining bei Pflegekräften und Ärzten

Mangelnde Fixierung/Sicherung des sedierten Patienten (Sturz!)

Keine Gewährleistung schneller Hilfe durch Anästhesie/Notfallteam

Keine systematische Erkennung und kein Management von Risikopatienten

Oben Genanntes gilt auch für die Überwachung nach dem Eingriff!

CIRS sind heute meist computer- und internetbasierte Softwaretools, die es allen Mitarbeitern erlauben, anonym und sanktionsfrei auf vorhandene (System-)Fehlerquellen und Missstände aufmerksam zu machen. Infolgedessen werden diese dokumentiert und für die restliche Belegschaft transparent. So muss derselbe «Fehler» nicht mehrfach gemacht werden - dies bedeutet Lernen von anderen und Lernen für andere (Beispiele siehe [27, 28]).

CIRS sollten durch eine professionelle Anonymisierung sowie Deidentifikation der Meldung garantieren, dass niemand die Originalmeldung kennt und dass eine personenbezogene Rückverfolgung nicht durchführbar ist. Darüber hinaus muss die Speicherung der Meldedaten maximalen Sicherheitsansprüchen genügen. Institutionsübergreifende CIRS genießen durch Information einer breiteren Fachöffentlichkeit den Vorteil, dem Presserecht zu unterliegen, und erfahren aus diesem Grund einen weitreichenden rechtlichen Datenschutz (Aussageverweigerungsrecht, Beschlagnahmeeinschränkung usw.) [29].

Herzstück jedes CIRS ist die zeitnahe und systematische Analyse (Fehlerwurzelsuche, Root Cause Analysis (RCA) [30, 31]) des gemeldeten Beitrags. Die systematische Fallanalyse wird von einem (externen) interdisziplinären und speziell geschulten Expertenteam bestehend aus Ärzten und Pflegepersonal durchgeführt. Bei Bedarf können Kooperationspartner, z.B. aus der Arbeits- und Organisationspsychologie, fallweise hinzugezogen werden. Der Leitungsebene werden sicherheitsrelevante Sachverhalte systematisch aufbereitet vorgestellt. Hierfür benötigt das CIRS-Team Arbeitszeit! Dies gewährleistet dann eine effektive Reaktion auf vorhandene Meldungen. Es gilt jedoch zu bedenken: Die Anhäufung von Rückmeldungen ohne konsekutiv umgesetzte Verbesserungsmaßnahmen führt leicht zur Frustration der meldenden Mitarbeiter und zu einem Vertrauensverlust in die Effektivität des Meldesystems.

Jede Meldung kann zu einer Optimierung der Systemsicherheit beitragen! Setzen Sie sich für die Planung und Umsetzung eines effektiven CIRS in Ihrer Abteilung und Klinik ein (Kasten 4). Falls Sie schon ein CIRS haben: Machen Sie mit! 
Tab. 4. Auszüge aus der S3-Leitlinie «Sedierung in der gastrointestinalen Endoskopie» (AWMF-Register-Nr. 021/014) [5]

\begin{tabular}{|c|c|}
\hline Patientenauswahl & $\begin{array}{l}\text { kritisches Sedierungsangebot/differenzierte Indikationsstellung von endoskopischen Eingriffen unter Prämedikation/ } \\
\text { (Analgo-)Sedierung (Empfehlungsgrad A, Evidenzstärke 5, § } 1.1 \text { und 1.2 [5]). Identifizierung von Risikopatienten (Tab. 3) } \\
\text { (Empfehlungsgrad A, Evidenzstärke 5, § 1.5 [5]). }\end{array}$ \\
\hline Patientenaufklärung & $\begin{array}{l}\text { Information der Patienten von ärztlicher Seite über die Nebenwirkungen/Komplikationen einer Sedierung bei } \\
\text { Endoskopien (Empfehlungsgrad A, Evidenzstärke } 5, \S 4.1[5] \text { ). }\end{array}$ \\
\hline Patientenvorbereitung & $\begin{array}{l}\text { Voraussetzung intravenöse Sedierung: sicherer und permanenter intravenöser Zugang (Empfehlungsgrad A, } \\
\text { Evidenzstärke 2b, § 4.2 [5]). Die Autoren empfehlen dringend, patientennahe Rückschlagventile und laufende Infusionen } \\
\text { zu verwenden. Prophylaktisch Sauerstoffgabe über eine Nasensonde bei sedierten Patienten (Empfehlungsgrad A, } \\
\text { Evidenzstärke 2b, § } 4.3[5] \text { ) vermindert Häufigkeit hypoxämischer Schäden signifikant }[32,33] \text {. }\end{array}$ \\
\hline $\begin{array}{l}\text { Ausbildung und } \\
\text { Training }\end{array}$ & $\begin{array}{l}\text { Qualitätssicherung: speziell auf die Sedierung ausgerichtetes Training für Ärzte und nichtärztliches Personal } \\
\text { (Empfehlungsgrad A, Evidenzstärke 4, § 3.2 [5]) inklusive Notfallausbildung und Beherrschung der kardiopulmonalen } \\
\text { Reanimation. Qualifikation durch periodische Teilnahme von ärztlichem und nichtärztlichem Personal an strukturierten, } \\
\text { gemeinsamen Fortbildungscurricula in theoretischen Kenntnissen und praktischen Fähigkeiten, inklusive Zwischenfalls- } \\
\text { management (z.B. Simulatormodel) (Empfehlungsgrad A, Evidenzstärke 5, § 3.3.1 [5]). Die Autoren empfehlen dringend, } \\
\text { moderne Teamtrainings durchzuführen. Nur das Training in dem Team, das später zusammen den Zwischenfall meistern } \\
\text { muss, bringt einen zuverlässigen Trainingserfolg («Train together who work together»). Einmal im Jahr sollte ein Notfall- } \\
\text { teamtraining vor Ort, in der Endoskopieeinheit durchgeführt werden. Solche «In-situ»-Trainings haben nachgewiesener- } \\
\text { maßen einen hohen Effekt, weil neben dem Teamtraining auch das gesamte System (Strukturen und Prozesse) für das } \\
\text { Notfallmanagement überprüft wird («System Probing»). Der das sedierende Medikament applizierende Arzt hat über } \\
\text { profunde Kenntnisse der verwendeten Medikamente und deren Nebenwirkungen zu verfügen, sollte intensivmedizinisch } \\
\text { erfahren sein und kardiopulmonale Reanimation, manuelle Beatmung, Sicherung der Atemwege sowie Intubation und } \\
\text { Kontrolle der Notfallsituation beherrschen (Evidenzgrad A, Evidenzstärke 4, § 3.1 [5]). Aus Sicht des Autors (M.R.) ist } \\
\text { diese Anforderung nach Inkrafttreten des Patientenrechtegesetzes noch nachhaltiger zu erfüllen (Qualifikationsmangel } \\
\text { bedeutet dort nämlich Beweislastumkehr!). }\end{array}$ \\
\hline
\end{tabular}

Medikamenten- $\quad$ Art/Intensität der Sedierung sowie die Wahl des Medikamentes in Abstimmung zu Art des Eingriffs, ASA-Klassifizierung applikation und Risikoprofil des Patienten sowie zur räumlichen, apparativen und personellen Ausstattung (Empfehlungsgrad A, Evidenzstärke 5, § 1.4.1 [5]). Die Autoren empfehlen dringend, Risikopatienten großzügig anderweitig versorgen zu lassen (in der Klinik oder unter Hinzuziehung der Anästhesie). Patientenindividuelle Dosisanpassung der Medikamente (Empfehlungsgrad B, Evidenzstärke 2b, § 2.4.3 [5]). Sedierungs- und/oder Analgesieverfahren durch Nicht-Anästhesisten dürfen planbar keinen Sedierungsgrad erreichen, bei dem lebenserhaltende Reflexe beeinträchtigt oder ausgeschaltet werden (§ 1.4.1 [5]). Sollte im Einzelfall ein Sedierungsgrad erreicht werden, bei dem lebenserhaltende Reflexe beeinträchtigt oder ausgeschaltet werden und der Eingriff fortgesetzt werden soll, ist ein Anästhesist hinzuzuziehen (§ 1.4.1 [5]).

Überwachung Untersucher und Endoskopieassistenz sind nur mittelbar in die Überwachung eingebunden. Überwachung der Vitalfunktionen unter Sedierung obliegt einer weiteren, entsprechend qualifizierten und nachweislich geschulten Person (Empfehlungsgrad A, Evidenzstärke 5, § 3.3.2 [5]) (Autoren: extrem wichtiger Punkt!). Bei Risikopatienten oder langwierigen/komplexen Eingriffen mit entsprechend tiefer Sedierung sollte hierfür ein entsprechend qualifizierter Arzt zugegen sein (Empfehlungsgrad A, Evidenzstärke 5, § 3.3.2 und 3.3.3 [5]). Bei Risikopatienten: Hinzuziehung eines Anästhesisten sollte erwogen werden (Evidenzgrad A, Evidenzstärke 5, § 1.5 [5]). Sedierte Patienten dürfen niemals unbeaufsichtigt sein. Die die Sedierung überwachende Person darf in dieser Zeit keine anderen Aufgaben wahrnehmen (Empfehlungsgrad A, Evidenzstärke 1b, § 3.3.3.1 [5]). Autoren: Dies muss auch gegen großen Widerstand in der Praxis durchgesetzt werden, vor allem bei Krankheit oder anderweitigem Personalmangel!

\begin{tabular}{|c|c|}
\hline Lagerung & $\begin{array}{l}\text { uf eine korrekte Lagerung sollte geachtet werden, um Lagerungsschäden zu vermeiden (§ } 1.7 \text { [5]). Die Patienten sollten } \\
\text { gemessen gesichert sein }(\S 1.7[5]) \text {. }\end{array}$ \\
\hline $\begin{array}{l}\text { Postendoskopische } \\
\text { Überwachung }\end{array}$ & $\begin{array}{l}\text { Nach Beendigung der Untersuchung müssen währenddessen sedierte Patienten von entsprechend geschultem und } \\
\text { qualifiziertem Personal in geeigneten Räumlichkeiten lückenlos überwacht werden (Empfehlungsgrad A, } \\
\text { Evidenzstärke 5, § 3.3.4 [5]). Autoren: «Lückenlos» ist zu gewährleisten! }\end{array}$ \\
\hline Räumliche Ausstattung & $\begin{array}{l}\text { Die Interventionsumgebung muss hinreichend zur Überwachung und Unterstützung der Atmungs- und Herz-Kreislauf- } \\
\text { Funktionen ausgestattet sein (Empfehlungsgrad A, Evidenzstärke 5, § } 3.4 \text { [5]). Ein zusätzlicher und separater Aufwach- } \\
\text { bereich sollte vorgehalten werden (Empfehlungsgrad A, Evidenzstärke 5, § } 3.4 \text { [5]). }\end{array}$ \\
\hline $\begin{array}{l}\text { Apparative und } \\
\text { medizinische } \\
\text { Ausstattung }\end{array}$ & $\begin{array}{l}\text { Monitoring im Behandlungsraum: Pulsoxymetrie, Blutdruck, EKG (Empfehlungsgrad A, Evidenzstärke 5, } \S 3.4 \text { und } \\
\text { 3.5 [5]). Ausstattung: Medikamente, Sauerstoffanschluss, Absaugung und Hilfsmittel/Gerätschaften für die Durchführung } \\
\text { der kardiopulmonalen Reanimation (Empfehlungsgrad A, Evidenzstärke 5, § } 3.4 \text { und 3.5 [5]). Sicherstellung einer } \\
\text { geeigneten Transportmöglichkeit in eine qualifizierte Behandlungseinrichtung (z.B. Intensivstation) (Empfehlungsgrad A, } \\
\text { Evidenzstärke 5, § } 3.4 \text { und 3.5 [5]). Autorenkommentar: Die Überwachung der Atemfrequenz ist sensibler als eine } \\
\text { alleinige Pulsoxymetrie. Die Pulsoxymetrie zeigt erst das Resultat einer Hypoventilation (keine Frühwarnung). } \\
\text { Die Atemfrequenz und der } \mathrm{CO}_{2} \text {-Wert (Kapnometrie auch bei Spontanatmung möglich) sind aber Frühindikatoren! }\end{array}$ \\
\hline
\end{tabular}


Um die Patientensicherheit in der Endoskopie nachhaltig zu erhöhen, müssen neben der Einhaltung der hier in Auszügen beschriebenen S3-Leitlinie (Tab. 4) die weiter oben aufgeführten Maßnahmen zur Erhöhung der Systemsicherheit ebenfalls in das Aus- und Weiterbildungskonzept aller Mitarbeiter integriert werden. Hierzu gehört einerseits die Verwendung von CIRS als auch insbesondere Schulungen im Bereich Fehlerentstehung, HFs und CRM sowie CRM-basierte Simulationsteamtrainings. Zwischenfälle können nur nachhaltig minimiert werden, wenn deren Hauptursachen im Bereich HFs effektiv geschult werden. Dies erfordert ein intensives, regelmäßiges (zweimal pro Jahr) und hoffentlich bald verpflichtendes Simulationsteamtraining für alle!
Marcus Rall ist Gründer und Geschäftsführer von InPASS, Institut für Patientensicherheit und Teamtraining GmbH. Bis August 2012 war er 17 Jahre an der Universitätsklinik Tübingen als Anästhesist und Notarzt tätig. Dort gründete er 1998 das Tübinger Patientensicherheits- und Simulationszentrum (TüPASS) und leitete es bis zu seinem Ausscheiden. InPASS bietet in Zusammenarbeit mit verschiedenen klinischen Partnern Dienstleistungen zu allen Bereichen der Patientensicherheit an. Hierzu gehören unter anderem Simulationsteamtrainings, Instruktorenkurse, HF-/CRM-Schulungen und die Beratung zu CIRS. CIRS-Training wird in Kooperation mit der Firma InWorks, Ulm, angeboten.

\section{Literatur}

1 Assmann A, Heinrichs W, Landauer B: Zusammenfassung der S3-Leitlinie «Sedierung in der gastrointestinalen Endoskopie». Anästh Intensivmed 2009;50:176-181.

- 2 Sieg A, Hachmoeller-Eisenbach U, Eisenbach T: Prospective evaluation of complications in outpatient GI endoscopy: a survey among German gastroenterologists. Gastrointest Endosc 2001;53:620-627.

- 3 Froehlich F, Gonvers JJ, Fried M: Conscious sedation, clinically relevant complications and monitoring of endoscopy: results of a nationwide survey in Switzerland. Endoscopy 1994:26:231-234.

4 Fruhmorgen P, Kriel L: Leitlinien zur endoskopischen Ektomie kolorektaler Polypen mit der Schlinge. Z Gastroenterol 1998;36:117-119.

5 Riphaus A, Wehrmann T, Weber B, et al: [S3guidelines - sedation in gastrointestinal endoscopy]. Z Gastroenterol 2008;46:1298-1330.

6 Riphaus A, Rabofski M, Wehrmann T: Sedierung in der gastrointestinalen Endoskopie in Deutschland. Z Gastroenterol 2010;48:392-397.

7 Rall M, Gaba DM: Human performance and patient safety; in Miller RD (ed): Miller's Anesthesia. Philadelphia, Elsevier, Churchhill Livingstone, 2009, pp 93-150.

8 Rall M, Manser T, Guggenberger H, Gaba DM, Unertl K: Patientensicherheit und Fehler in der Medizin. Entstehung, Prävention und Analyse von Zwischenfällen. Anästhesiol Intensivmed Notfallmed Schmerzther 2001;36:321-330.

9 Reason JT: Human Error. Cambridge, Cambridge University Press, 1990.

10 Reason JT, Carthey J, de Leval MR: Diagnosing 'vulnerable system syndrome': an essential prerequisite to effective risk management. Qual Health Care 2001;10(suppl 2):ii21-ii25.

11 Dismukes K, Berman BA, Loukopoulos L: The Limits of Expertise - Rethinking Pilot Error and the Cause of Airline Accidents. Aldershot, Ashgate, 2007.
12 Reason J: Managing the Risks of Organizational Accidents. Aldershot, Ashgate, 1997.

13 Aken HV, Biermann E, Martin J, et al: Analgosedierung für diagnostische und therapeutische Maßnahmen bei Erwachsenen. Anästh Intensivmed 2010;51:598-602.

14 Roberts K: Some characteristics of high reliability organizations. Organ Sci 1990;1:160-177.

15 Roberts K, Rousseau D, LaPorte T: The culture of high reliability: quantitative and qualitative assessment aboard nuclear powered aircraft carriers. J High Technol Manage Res 1994;5:141-161.

16 Hollnagel EW, David D, Leveson N: Resilience Engineering - Concepts and Precepts. Aldershot, Ashgate, 2006.

17 Wiener EL, Kanki BG, Helmreich R (eds): Cockpit Resource Management. San Diego, Academic Press, 1993.

18 Hicks CM, Bandiera GW, Denny CJ: Building a simulation-based crisis resource management course for emergency medicine, phase 1: Results from an interdisciplinary needs assessment survey. Acad Emerg Med 2008;15:1136-1143.

19 Issenberg SB, Scalese RJ: Simulation in health care education. Perspect Biol Med 2008;51:31-46.

20 Rall M, Dieckmann P: Safety culture and crisis resource management in airway management: general principles to enhance patient safety in critical airway situations. Best Pract Res Clin Anaesthesiol 2005;19:539-557.

21 Rall M, Gaba DM: Patient simulation; in Miller RD (ed): Miller's Anesthesia, ed 7. Philadelphia, Elsevier, Churchhill Livingstone, 2009, pp 151-192.

22 Berkenstadt H, Erez D, Munz Y, Simon D, Ziv A: Training and assessment of trauma management: the role of simulation-based medical education. Anesthesiol Clin 2007;25:65-74, viii-ix.
23 Rudolph JW, Simon R, Raemer DB, Eppich WJ: Debriefing as formative assessment: closing performance gaps in medical education. Acad Emerg Med 2008;15:1010-1016.

24 Kohn LT, Corrigan JM, Donaldson MS: To Err Is Human: Building a Safer Health System. Washington, National Academy Press, 2000.

25 www.arztbibliothek.de/themenschwerpunkt/patientensicherheit.

26 www.aktionsbuendnis-patientensicherheit.de/apsside 07-07-25-CIRS-Handlungsempfehlung.pdf.

27 www.cirsmedical.de.

$28 w w w$.cirshealthcare.de

29 Van Aken HL: PaSOS - ein entscheidender Schritt zur Erhöhung der Patientensicherheit in der Anästhesie. Das Patienten-Sicherheits-OptimierungsSystem PaSOS - über 700 Fälle, transparent, sicher, neu mit einzelfallbezogenem Feedback und Analyseoption. Anaesth Intensivmed 2008;49:246.

30 Bagian JP, Gosbee J, Lee CZ, Williams L, McKnight SD, Mannos DM: The Veterans Affairs root cause analysis system in action. Jt Comm J Qual Improv 2002;28:531-545.

31 National Patient Safety Agency (NPSA): Root cause analysis RCA toolkit. www.npsa.nhs.uk/ health/resources/root_cause_analysis/conditions.

32 Jurell KR, O'Connor KW, Slack J, Fraiz J, Shaar CJ, Kent L, Callon R: Effect of supplemental oxygen on cardiopulmonary changes during gastrointestinal endoscopy. Gastrointest Endosc 1994; 40:665-670.

33 Reshef R, Shiller M, Kinberg R, Rennert H, Rennert G, Herskovits M, Loberant N: A prospective study evaluating the usefulness of continuous supplemental oxygen in various endoscopic procedures. Isr J Med Sci 1996;32:736-740. 